\title{
Cardiologia Baseada em Evidências - IV. Principais Estratégias de Pesquisa e Níveis de Recomendações em Cardiologia
}

\author{
Álvaro Avezum \\ São Paulo, SP
}

\section{Introdução}

O paradigma do processo científico é a observação controlada adquirida através do experimento, enquanto que o paradigma da pesquisa epidemiológica não-experimental é o "experimento natural". Quando experimentos não são factíveis, estudos não-experimentais são planejados para simular o que poderia ser aprendido se um experimento tivesse sido conduzido. A investigação clínica científica quase sempre considera que um grupo controle seja utilizado para que a nova intervenção possa ser comparada e a randomização é a maneira preferida de designar pacientes para os grupos controle e intervenção. Em estudos experimentais, a intervenção em questão encontra-se sob o controle do investigador, por exemplo, o estudo clínico controlado randomizado. Em contrapartida, quando se trata de estudos observacionais, a intervenção em questão não se encontra sob controle do investigador, por exemplo, estudo caso-controle.

A estratégia de obtenção dos dados para um estudo pode ser prospectiva ou retrospectiva. Em estudos retrospectivos, a definição da doença, dos sintomas e das unidades de medidas podem ter sido modificadas com o passar do tempo, como também os métodos diagnósticos podem ter sido substituídos, resultando assim, em maior variabilidade dos dados. A grande vantagem dos estudos prospectivos é que permitem a determinação da direção dos eventos (o que aconteceu antes e o que aconteceu depois) e também, que a natureza dos dados (diagnóstico, definições, etc.) apresenta pouca variabilidade. A comparação dos grupos pode ser histórica (comparação dos dados obtidos com estudos anteriores) ou contemporânea (comparação dos dados obtidos com estudos recentes) ${ }^{1,2}$.

As principais estratégias de pesquisa podem ser divididas em descritivas/analíticas e experimentais. Discutiremos no grupo de estratégia descritiva/analítica os seguintes estudos: relato de série, transversal, caso-controle e de coorte. No

Instituto Dante Pazzanese de Cardiologia

Correspondência: Álvaro Avezum - Instituto Dante Pazzanese de Cardiologia Av. Dante Pazzanese, 500 - $12^{\circ}$ - 04012-180 - São Paulo, SP

Recebido para publicação em 22/8/98

Aceito em 22/9/98 grupo de estratégia experimental discutiremos estudo controlado randomizado e estudo randomizado cruzado.

\section{Estratégias descritivas/analíticas}

Estes planejamentos de estudos são mais apropriados quando o experimento nãoé factível em relação à variável em questão, como por exemplo, a exposição a agentes potencialmente prejudiciais (tabagismo) ou situações nas quais pode haver um longo intervalo entre a exposição e a ocorrência do evento. $\mathrm{O}$ investigador não controla as circunstâncias de exposição. Dentro destas estratégias de pesquisa, o investigador dependerá fortemente da seleção de indivíduos para o estudo, no que diz respeito ao esclarecimento da questão formulada.

\section{Estudo relato de série}

Característica principal - Descrição de uma série de casos, para ilustrar um aspecto de uma determinada condição clínica, sem utilizar um grupo controle para comparação. Deve ser interpretado apenas como informações preliminares que devem ser avaliadas apropriadamente em investigações posteriores através de estudos bem planejados.

Vantagens - 1) Descrição de um novo método diagnóstico, intervenção clínica ou procedimento cirúrgico;2) pode fornecer uma grande quantidade de informações sobre uma condição clínica.

Desvantagens - 1) Não existe comparação com controle, não sendo possível avaliar a eficácia real de intervenções terapêuticas ou acurácia de testes diagnósticos; 2) geralmente, descreve as manifestações clínicas da doença e seus tratamentos em um grupo de pacientes constituído em um ponto no tempo; 3 ) valor restrito em estudar relações prognósticas ou de causa e efeito ${ }^{2,3}$.

\section{Estudo transversal (prevalência)}

Característica principal-Uma amostra representativa da população é entrevistada, examinada ou estudada para se obter resposta para uma questão específica. Os gru- 


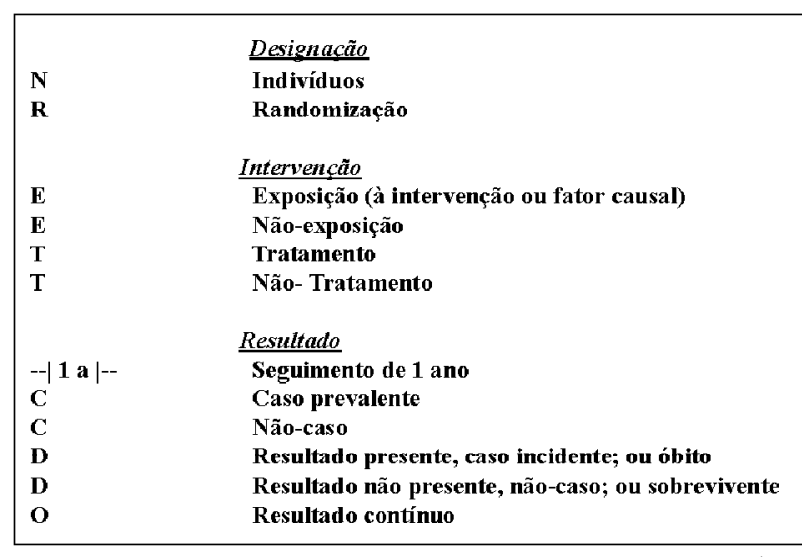

Fig. 1 - Nomenclatura para estratégias de pesquisa epidemiológica (adaptado ${ }^{1}$ )

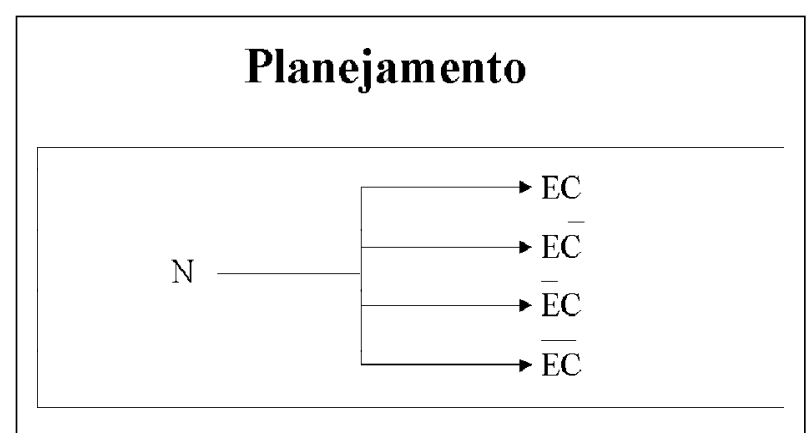

Fig. 2 -Estudo transversal (adaptado ${ }^{1}$ )

pos sob exposição e controle são determinados simultaneamente (fig. 1 e 2).

Vantagens - 1) Execução simples; 2) custos relativamente baixos; 3) não é necessário seguimento; 4) os indivíduos não são expostos ao agente causal devido ao estudo ou deixa de receber um potencial benefício do tratamento em avaliação; 5) avaliação de fatores de risco; 6) avaliação de prevalência de doenças.

Desvantagens -1) Pode estabelecer associação, mas não causalidade; 2) impossível de se assegurar que fatores de confusão estejam igualmente distribuídos entre os grupos; 3 ) os grupos podem apresentar tamanhos de amostras diferentes, resultando em perda de eficiência estatística; 4) não avalia história natural ou prognóstico de doenças; 5) suscetível do viés de incidência/prevalência ${ }^{1-4}$.

\section{Estudo caso-controle}

Característica principal - Os grupos são identificados com base nos resultados clínicos (por exemplo: infarto do miocárdio) e a busca pela exposição (por exemplo: tabagismo, contraceptivos orais, etc.) é retrospectiva. Os pacientes (casos) são emparelhados com controles (pacientes com outro tipo de doença, população geral, vizinhos ou parentes) (fig. 3).

Vantagens - 1) Pode ser realizado em tempo relativamente curto; 2) custos relativamente baixos; 3) pode ser o único método para avaliar doença raras ou situações onde

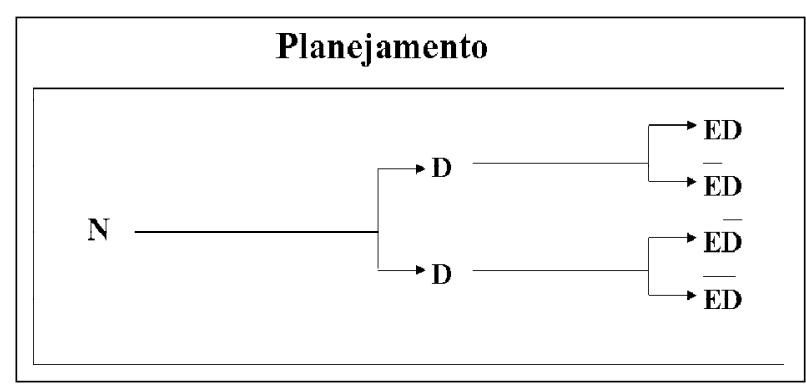

Fig. 3 - Estudo caso controle (adaptado ${ }^{1}$ )

existe um grande intervalo entre exposição e evento; 4) geralmente, requer menos pacientes do que estudos transversais; 5) formular hipóteses para futuros estudos.

Desvantagens - 1) Depende da lembrança do paciente ou dos registros para determinar a exposição, os quais notoriamente não são acurados; 2) os grupos podem apresentar fatores de confusão (o evento pode ter sido causado por outro fator que não a exposição em investigação; 3) a identificação de um grupo controle adequado pode ser difícil; 4) não pode demonstrar causalidade (a associação entre fator e resultado clínico não prova necessariamente que fator causou o resultado clínico); 5) possibilidade de viéses de seleção e de aferição ${ }^{1-4}$.

\section{Estudo de coorte (seguimento)}

Característica principal - Os pacientes ou indivíduos são divididos entre expostos (ou tratados) e não-expostos (ou não-tratados), com base na história pregressa e seguidos por um período para avaliar quantos em cada grupo desenvolveram uma doença particular ou um resultado clínico. Os indivíduos são livres de doença no início, diferem de acordo com a extensão da exposição a uma causa potencial da doença e são comparados com relação à incidência da doença em cada grupo. O planejamento pode ser prospectivo ou retrospectivo. Quando tanto à exposição quanto à doença são históricos, o estudo é descrito como coorte retrospectivo, seguimento retrospectivo ou coorte histórico. Os custos de um estudo coorte retrospectivo são menores do que um coorte prospectivo (fig. 4).

Vantagens - 1) O tratamento não é deixado de ser oferecido aos pacientes (os quais não ficam sujeitos a riscos potenciais); 2) os pacientes podem ser parelhados para

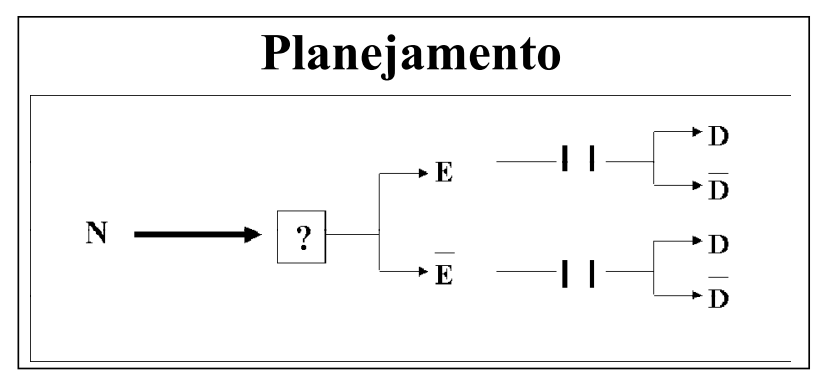

Fig. 4 - Estudo de coorte (adaptado ${ }^{1}$ ) 
possíveis e potenciais fatores de confusão; 3 ) quando prospectivo, os critérios de inclusão e exclusão, como também a avaliação dos resultados clínicos, podem ser padronizados; 4) pode estabelecer o tempo de ocorrência (temporalidade) e a direção dos eventos.

Desvantagens - 1) A obtenção de controles pode ser difícil se o tratamento for relativamente difundido, ou se a maioria dos indivíduos tiver sido exposta; 2) a exposição pode estar relacionada a outros fatores não conhecidos que podem estar correlacionados com o resultado clínico (fatores de confusão); 3) a condição cega entre pacientes e pesquisadores pode ser difícil de ser obtida; 4) quando bem realizado, torna-se de custo alto; 5) no caso de doenças raras, torna-se necessário um seguimento maior, como também maior tamanho de amostra ${ }^{1-4}$.

Estratégias experimentais - A intervenção encontra-se sob o controle do investigador, ou seja, através do investigador houve a designação aleatória ou randomizada para a estratégia em questão. Estas estratégias são utilizadas em estudos avaliando terapia, devido ao fato de serem menos suscetíveis aos vários riscos à validade, como por exemplo, viéses.

\section{Estudo controlado randomizado}

Característica principal - A designação de pacientes para os tratamentos ou exposições encontra-se sob o controle do investigador. Os pacientes serão designados de maneira randomizada para qualquer uma das intervenções sob estudo. Ambos os grupos são seguidos por um período de tempo específico e analisados em termos de resultados clínicos definidos pelo protocolo do estudo (exemplo: óbito, reinfarto, hospitalização por insuficiência cardíaca, etc.) (fig. 5).

Vantagens - 1) Os grupos apresentam maior probabilidade de comparação porque os fatores de confusão encontram-se provavelmente balanceados entre os grupos; 2 ) há maior probabilidade do pesquisador e pessoal envolvidos no estudo estarem sob a condição cega; 3) a maioria dos testes estatísticos está vinculada ao princípio da designação randomizada; 4) permite avaliação rigorosa de efeito

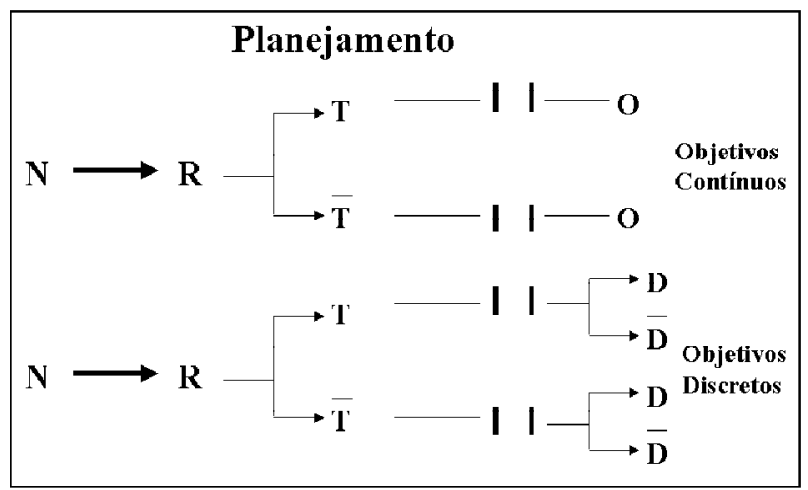

Fig. 5 - Estudo controlado randomizado (adaptado ${ }^{1}$ ) de uma droga versus placebo ou outra droga em uma grupo precisamente definido (exemplo: infarto agudo do miocárdio dentro das primeiras $12 \mathrm{~h}$ de início dos sintomas em pacientes com mais de 50 anos de idade); 5) planejamento prospectivo; 6) erradicação potencial de viéses; 7) permite utilização para revisões sistemáticas ou metanálises.

Desvantagens - 1) Custos mais elevados e maior consumo de tempo; 2) os pacientes que voluntariamente aceitam participar do estudo podem não ser representativos da população; 3) segundo alguns, um tratamento potencialmente efetivo não seria administrado a alguns pacientes. Entretanto, desde que existam dúvidas quanto à eficácia de um tratamento, a melhor maneira de esclarecer e confirmar as sugestões promissoras é a comprovação científica através do estudo clínico controlado randomizado; 4) algumas vezes utiliza-se objetivos substitutos ao invés de objetivos clínicos relevantes preferencialmente; 5) falha em randomizar todos pacientes que preencham os critérios de inclusão e em aplicar a condição cega para os investigadores ${ }^{1-4}$.

\section{Estudo randomizado cruzado}

Característica principal-Cada paciente recebe tratamento ativo e controle de maneira randomizada, separado por um período de wash-outsem nenhum tratamento. Como todos os pacientes receberão tratamento ativo e controle, este planejamento permite que os pacientes sejam controles de si mesmos (fig. 6).

Vantagens -1) Os pacientes são seus próprios controles, reduzindo assim a variância e conseqüentemente o tamanho da amostra (menos pacientes do que em um estudo controlado randomizado); 2) todos os pacientes recebem tratamento ativo e placebo pelo menos por um período; 3 ) testes estatísticos assumindo designação randomizado podem ser utilizados; 4) a condição cega do pesquisador e do pessoal envolvido no estudo pode ser estabelecida.

Desvantagens -1) Pacientes que respondem ao tratamento são retirados do mesmo e placebo ou tratamento pa-

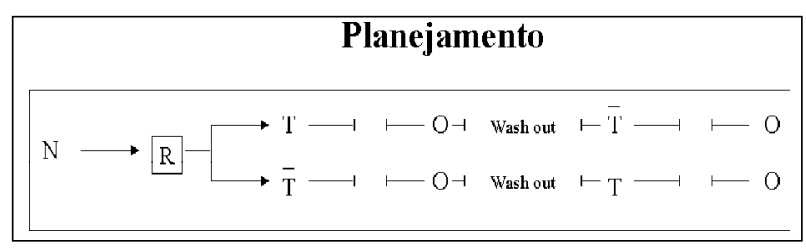

Fig. 6 - Estudo randomizado cruzado (adaptado ${ }^{1}$ )

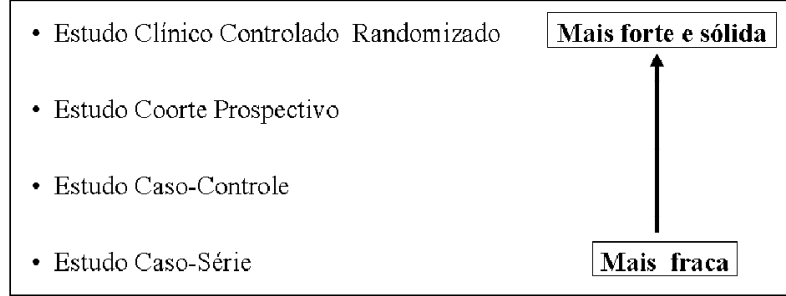

Fig. 7 - Qualidade da evidência 
drão. Entretanto, se existem dúvidas quanto à eficácia, o estudo deve prosseguir; 2) o período de eliminação do efeito da droga pode ser relativamente longo, durante o qual paciente receberia placebo; 3 ) não pode ser utilizado se o tratamento apresentar qualquer efeito permanente (por exemplo: programas educacionais, fisioterapia, ou terapia comportamental) ${ }^{1-3}$.

\section{Graduação de estratégias de pesquisa e roteiro de recomendações utilizados na cardiologia baseada em evidências}

As recomendações, principalmente envolvendo intervenções terapêuticas devem ser graduadas para que possamos colocar as informações provenientes da pesquisa clínica dentro de uma perspectiva de evidências científicas. Quanto menor possibilidade de erros sistemáticos, erros aleatórios, fatores de confusão, contaminação e co-intervenção, melhor a qualidade metodológica do estudo em questão. A arquitetura das informações provenientes das diferentes estratégias de pesquisa devem ser examinadas para verificação de consistência, coerência e clareza. Através da utilização deste roteiro de recomendações baseadas em evidências, a decisão clínica torna-se embasada em uma prática médica objetiva, adequada e cientificamente correta no que concerne ao tratamento de nossos pacientes.

\section{Grau A}

Nível 1a - Evidência de estudos randomizados amplos (ERAs) ou revisões sistemáticas (incluindo metanálises) de vários estudos randomizados, os quais tenham tantos dados como um estudo bem definido.

Nível 1b-Evidência de pelo menos um estudo de coorte de alta qualidade, nos quais todos os pacientes faleceram/ sem sucesso com terapia convencional e alguns sobreviveram/com sucesso com a nova terapia (ex: desfibrilação para fibrilação ventricular); ou nos quais muitos faleceram/ sem sucesso com a terapia convencional e nenhum faleceu/ com sucesso com a nova terapia (ex: penicilina para infecções pneumocócicas).

Nível 1c - Evidência de pelo menos um ERA de tamanho moderado ou uma revisão sistemática (ou metanálise) de pequenos estudos, os quais coletivamente apresentam um número moderado de pacientes.

Nível 1d - Evidência de pelo menos um ERA.

\section{Grau B}

Nível 2 - Evidência de pelo menos um estudo de coortes não-randomizado que recebeu e não recebeu a nova terapia.

Nível 3 - Evidência de pelo menos um estudo casocontrole de alta qualidade.

Nível 4 - Evidência de pelo menos um estudo de relato de série de alta qualidade.

\section{Grau C}

Nível 5 - Opiniões de peritos sem referência ou acesso a qualquer um dos estudos anteriores (i.e.: argumentos fisiológicos, experiência pessoal ou princípios básicos) ${ }^{5}$.

\section{Considerações finais}

A escolha do planejamento do estudo ou estratégia de pesquisa clínica depende basicamente da questão enunciada. Geralmente, várias estratégias são possíveis e factíveis, contudo devemos escolher aquela considerada de qualidade de evidência científica mais forte, sólida, confiável e robusta, ou seja, que permita menos alternativas explanatórias para os resultados obtidos. Como pudemos observar, existe uma hierarquia de evidências científicas que deve ser respeitada e seguida para que nossa decisão clínica seja correta, ou seja, fazer o correto de maneira correta (fig. 7). Dentro da área de terapia em cardiologia sugerimos a seguinte interpretação e utilização das diferentes estratégias de pesquisa: 1) identificar áreas promissoras de pesquisa: a) investigações laboratoriais (pesquisa básica); b) relato de série; c) estudo caso-controle; d) estudo de coorte; 2) gerar hipóteses para serem testadas posteriormente: a) estudos randomizados pequenos/moderados; b) revisões sistemáticas de pequenos/moderados estudos; 3 ) testar hipóteses para decisão clínica e recomendações: a) estudos randomizados amplos com poder estatístico adequado; b) revisões sistemáticas de estudos randomizados amplos.

\section{Referências}

1. Streiner DL, Norman GR, Blum HM - PDQ Epidemiology. Toronto: BC Decher, 1989: 45-52.

2. Avezum A - Cardiologia baseada em evidências e avaliação crítica da literatura cardiológica: princípios de epidemiologia clínica aplicados à Cardiologia. Soc Cardiol ESP 1996; 6: 241-59.
3. Fletcher RH, Fletcher SW, Wagner EH - Clinical Epidemiology - The Essentials $3^{\text {th }}$ ed. Baltimore: Williams \& Wilkins, 1996: 208-27.

4. Rothman KJ - Modern Epidemiology. Boston/Toronto: Little, Bronw, 1986: 51-76

5. Kitching A, Sackett D, Yusuf S - Approaches to evaluating evidence. Evidence Based Cardiology. London: BMJ Books, 1998: 3-23. 